\title{
MICROALGAS COMO MATÉRIA-PRIMA NA PRODUÇÃO DE BIODIESEL: UMA REVISÃO
}

\section{MICROALGAE AS RAW MATERIAL FOR BIODIESEL PRODUCTION: A REVIEW}

\section{R.P. CRUZ ${ }^{1}$, B.L.S. SANTIAGO ${ }^{1}$ and E.L. PEREIRA ${ }^{2}$}

${ }^{1}$ Federal University of Viçosa, Department of Chemistry, Viçosa, Minas Gerais, Brazil, rodolfo_pinheiro@hotmail.com, blsilvasa@gmail.com

${ }^{2}$ Federal University of Ceará, Department of Hydraulic and Environmental Engineering, Fortaleza, Ceará, Brazil, erlonlopes@gmail.com

\begin{tabular}{|c|}
\hline A R T I CLE INFO \\
\hline $\begin{array}{l}\text { Article history: } \\
\text { Received 2019-02-16 } \\
\text { Accepted 2019-06-27 } \\
\text { Available online 2019-06-30 }\end{array}$ \\
\hline $\begin{array}{l}\text { palavras-chave } \\
\text { Biocombustível } \\
\text { Biodiesel } \\
\text { Microalgas } \\
\text { Tecnologias ambientais } \\
\text { Processos biotecnológicos }\end{array}$ \\
\hline $\begin{array}{l}\text { keywords} \\
\text { Biofuel } \\
\text { Biodiesel } \\
\text { Microalgae } \\
\text { Environmental technology } \\
\text { Biotechnology }\end{array}$ \\
\hline
\end{tabular}

\begin{abstract}
A B S T R A C T
Growing environmental concern has strengthened the search for renewable fuels, and biodiesel is especially advantageous because it has high energy density and allows replacement of the diesel. The production of biodiesel is still costly, and the raw material strongly affects the final price. Given the background, microalgae is a promising alternative, because of its advantages such as the ability to accumulate lipids, high growth rate, higher absorption of carbon dioxide, non-competition with arable crops for arable land, and the possibility of use in the treatment of effluents. In this context, the present review addresses the advantages and challenges of using microalgae as a raw material in biodiesel production, as well as the production technologies available on a large scale, its stages, and future improvement.
\end{abstract}

R E S U M O

A crescente preocupação ambiental tem fortalecido a busca por combustíveis renováveis. $O$ biodiesel é especialmente vantajoso por ter alta densidade energética e permitir substituição do diesel. A produção de biodiesel ainda é extremamente dispendiosa, e esse custo é fortemente afetado pela matéria prima. Neste contexto as microalgas são uma alternativa promissora, e ainda tem como vantagens a alta capacidade de acúmulo de lipídios, alta taxa de crescimento, maior absorção de gás carbônico, não competição com culturas alimentícias por terras aráveis e possibilidade de uso no tratamento de efluentes. Por conseguinte, a presente revisão aborda as vantagens e os desafios do emprego de microalgas como matériaprima na produção de biodiesel, assim como as etapas das tecnologias de produção disponíveis em grande escala e os avanços futuros. 


\section{INTRODUÇ̃̃O}

Nos últimos anos, a procura por combustíveis renováveis tem aumentado, seja pelas variações do preço do petróleo no mercado internacional ou pela preocupação com o meio ambiente (HALIM et al., 2012). Dentre os combustíveis renováveis viáveis tecnicamente, destaca-se o biodiesel, que tem vantagens ambientais, econômicas, sociais e tecnológicas.

O biodiesel é definido, pela Agência Nacional do Petróleo, Gás Natural e Biocombustíveis (ANP, 2012), como combustível composto por alquil-ésteres de ácidos carboxílicos de cadeia longa. Sua produção ocorre a partir da transesterificação e/ou esterificação de ácidos graxos, de gorduras de origem vegetal ou animal que respeite a especificação contida no Regulamento Técnico no 4/2012 da Resolução ANP No 14, de 11/5/2012.

Do ponto de vista ambiental o biodiesel é uma fonte de energia renovável, não tóxico, livre de compostos aromáticos e sulfurosos, e reduz a emissão de compostos como $\mathrm{SO}_{2}, \mathrm{CO}, \mathrm{CO}_{2}$ e particulados. A análise do ciclo completo do biodiesel indica uma redução de até $78 \%$ das emissões de dióxido de carbono (DOSHI et al., 2016). Do ponto de vista econômico, ele pode reduzir a necessidades de importações, gerar receitas com exportações, fortalecer o agronegócio, criar empregos na zona rural e aumentar a segurança energética do país (ANUAR e ABDULLAH, 2013). O biodiesel pode ser produzido a partir de diferentes fontes, como óleos vegetais (soja, palma, girassol, algodão, amendoim e outros), gordura animal e pelo reuso de óleo de fritura (VERMA e SHARMA, 2016). No entanto, a procura por novas fontes para a produção de biocombustíveis não se limita à óleos vegetais e à gordura animal, mas também à microalgas. De acordo com Pereira et al. (2012), as vantagens das microalgas são: a facilidade de cultivo; a quantidade acumulada de lipídeos, que pode chegar a $80 \%$ da massa seca; menor necessidade de nutrientes e água em relação às culturas terrestres; grande viabilidade de manipulação genética; alta velocidade de crescimento da biomassa; possibilidade de controlar as condições de cultivo. Além disso, elas não precisam de solos aráveis; podem ser utilizadas no tratamento de águas residuais, na biorremediação de metais pesados, nitrogênio e fósforo, que poderiam causar a eutrofização de cursos de água caso fossem descartados sem tratamento; e ainda podem auxiliar na mitigação de emissões de gás carbônico (ASSEMANY et al., 2016).

As microalgas são consideradas a única alternativa capaz de tornar o biodiesel viável para substituir completamente os combustíveis de petróleo. Segundo Chisti (2007), para atender o consumo anual nos Estados Unidos seriam necessários 0,53 bilhões de litros de biodiesel por ano, o que corresponde a ocupar $61 \%$ das áreas cultiváveis dos EUA com a cultura terrestre de maior produtividade (óleo de palma). Essa possibilidade é inviável, uma vez que não restaria terra suficiente para a produção de alimentos. Porém, caso a fonte de matéria-prima fosse o óleo proveniente das microalgas, apenas $3 \%$ da área agricultável seria ocupada (Chisti, 2007). A produção de biodiesel a partir de microalgas é centrada na produção de óleo, que será esterificado para a produção deste biocombustível. As etapas de produção são: cultivo da biomassa de microalgas e o seu processamento, que é composto pelas etapas de colheita, secagem e extração do óleo. O presente trabalho tem o objetivo de descrever detalhadamente os aspectos que envolvem cada uma das etapas da produção biotecnológica de óleo de microalgas, que será empregado na viabilização técnica da produção de biodiesel.

\section{MICROALGAS}

Microalgas são microrganismos unicelulares fotossintéticos que compõem o fito plâncton (Li et al., 2008). Estão presentes em todas as regiões do mundo em quase todos os ambientes aquáticos, salinos ou de água doce. São encontradas como células isoladas, formando colônias sob a forma de segmentos lineares de células. Em qualquer um dos arranjos não ocorre nenhuma diferenciação celular, ou seja, cada célula é responsável por todas as funções vitais (MATA et al., 2010). Elas podem ser procarióticas (cianobactérias) ou eucarióticas, estas classificadas em algas verdes (Chlorophyta), algas vermelhas (Rhodophyta) e diatomáceas (Bacillariophta).

Atualmente, as microalgas são consideradas a fonte mais promissora de biomassa para a produção de biodiesel (BORUGADDA e GOUD, 2012). Chisti (2007) afirma que as microalgas apresentam vantagens, que provêm de aspectos fisiológicos e bioquímicos, sobre as culturas terrestres. Primeiramente, a eficiência da conversão da luz solar durante a fotossíntese nas microalgas é de cerca de 6 a 12 vezes superior, o que permite que elas cresçam mais rápido em relação às culturas terrestres. A biomassa de microalgas duplica a cada 24 horas, e em algumas espécies esse tempo pode cair para até cerca de 3,5 horas durante a fase exponencial de crescimento (CHISTI, 2007). Particularidades de cada espécie afetam também a produtividade de óleo, que é uma característica singular, como pode ser observado na Tabela 1. Franco et al. (2013) explicam que o conteúdo de óleo por porcentagem de matéria seca encontrado em diferentes espécies de algas pode aumentar através da alteração das condições de cultivo e stress ambiental. De acordo com os autores, condições como a deficiência de nutrientes e anaerobiose são aplicadas estrategicamente em cultivos para aumentar a produção de lipídeos.

As estruturas de suporte da célula, formadas por polímeros nas células de biomassa vegetal por exemplo, não estão presentes nas microalgas, o que facilita a extração do óleo, aumentando a eficiência da conversão da biomassa em biocombustíveis e reduzindo os custos industriais (LYNDT et al., 2008). Além disso, a enorme diversidade ecológica, morfológica, metabólica e genética de microalgas existentes permite a escolha da espécie mais adequada as condições de cultivo e sistema de colheita adotado (DISMUKES et al., 2008). Patil et al. (2008) mencionam que as microalgas não necessitam de terras férteis para o seu cultivo, o que elimina a competição por áreas agricultáveis que assim podem ser usadas no cultivo de alimentos, o que é uma grande vantagem das microalgas em relação às culturas comumente usadas como matéria-prima para a produção de óleo. Outro fator importante que pode ser observado na Tabela 2, é que o conteúdo de óleo por porcentagem de matéria em seca encontrado nas microalgas é maior do que o encontrado nas culturas terrestres.

A produtividade de óleo não é o único fator que deve ser levado em conta para a escolha da melhor espécie de microalga para a produção de biodiesel. É importante observar fatores como: condições de crescimento; requisitos para a coleta, extração e purificação do óleo; resistência ao ataque de cepas selvagens não produtoras; composição em ácidos graxos do 
óleo extraído; possibilidade de uso como matéria-prima para a produção de outros produtos nas indústrias de alimentos, cosméticos e de fármacos (JIANG et al. 2011). A composição dos ácidos graxos é extremamente importante porque ela influencia as propriedades do biodiesel produzido, como a densidade, viscosidade, resistência à oxidação e ponto de fusão.
Essa composição varia com a espécie da microalga, e depende do número de carbonos na cadeia e presença de insaturações (FRANCO et al., 2013). Após a escolha da espécie de microalga mais adequada, a produção de biodiesel segue para a etapa de cultivo, que depende do meio de cultura e da forma de cultivo, como será detalhado a seguir.

Tabela 1 - Conteúdo e produtividade de óleo de algumas espécies de microalgas.

\begin{tabular}{lcc}
\hline \multicolumn{1}{c}{ Espécies de microalgas } & Conteúdo de óleo por \% da matéria seca & Produtividade de óleo $\left(\mathrm{mg} \mathrm{L}^{-1} \mathrm{~d}^{-1}\right)$ \\
\hline Nannochloropsis oculata & $22-30$ & $84-142$ \\
Chlorella vulgaris & $5-50$ & - \\
Dunaliella salina & $14-20$ & 116 \\
Neochloris oleoabundans & $35-65$ & $90-134$ \\
Chlorella sorokiniana & $19-22$ & 45 \\
Phaeodactylum tricornutum & $18-57$ & 45 \\
Spirulina máxima & $4-9$ & - \\
Chlorococcum sp. & 19 & 54 \\
Chlorella protothecoides & $23-55$ & 1214
\end{tabular}

Fonte: Mata et al. (2010)

Tabela 2 - Comparação entre as microalgas e outras fontes de óleo para fabricação de biodiesel.

\begin{tabular}{lcccc}
\multicolumn{1}{c}{ Fonte de Óleo } & $\begin{array}{c}\text { Conteúdo de óleo } \\
\text { por \% matéria em } \\
\text { seca }\end{array}$ & $\begin{array}{c}\text { Produção de Óleo } \\
\left(\text { L óleo há }^{-1} \text { ano }^{-1}\right)\end{array}$ & $\begin{array}{c}\text { Uso da terra }\left(\mathrm{m}^{2} \mathrm{~kg}\right. \\
\left.\text { biodiesel }^{-1} \mathrm{ano}^{-1}\right)\end{array}$ & $\begin{array}{c}\text { Produtividade de } \\
\text { biodiesel }\left(\mathrm{kg}^{-1}\right. \\
\left.\text { biodiesel ha }^{-1} \mathrm{ano}^{-1}\right)\end{array}$ \\
Milho & 44 & 172 & 66 & 152 \\
Cânhamo & 33 & 363 & 31 & 321 \\
Soja & 18 & 636 & 18 & 562 \\
Pinhão-Manso & 28 & 741 & 15 & 656 \\
Camelina & 42 & 915 & 12 & 809 \\
Canola & 41 & 974 & 12 & 862 \\
Girassol & 40 & 1070 & 11 & 946 \\
Mamona & 48 & 1307 & 9 & 1156 \\
Óleo de Palma & 36 & 5366 & 2 & 4747 \\
Microalga (baixo teor de óleo) & 30 & 58700 & 0.2 & 51927 \\
Microalga (médio teor de óleo) & 50 & 97800 & 0.1 & 86515 \\
Microalga (alto teor de óleo) & 70 & 136900 & 0.1 & 121104
\end{tabular}

Fonte: Mata et al. (2010)

\section{CULTIVO}

\subsection{Meio de Cultura}

O cultivo de microalgas requer um meio que forneça três macronutrientes: nitrogênio, fósforo e carbono. A fonte de carbono, especificamente, depende do tipo de metabolismo da microalgas que é próprio de cada espécie e pode ser: autotrófico, no qual o $\mathrm{CO}_{2}$ é a única fonte de carbono e a luz é convertida em energia química por meio da fotossíntese; heterotrófico que utiliza compostos orgânicos dissolvidos como fonte de energia e carbono; e mixotrófico que realiza fotossíntese e necessita de $\mathrm{CO}_{2}$ e fontes orgânicas de carbono para o seu crescimento (BRENNAN e OWENDE 2010, CHEN et al. 2011).

Também são necessários micronutrientes como sílica, cálcio, magnésio, potássio, ferro, zinco, cobalto, cobre, manganês e enxofre (CHRISTENSON e SIMS, 2011). Amaro et al. (2011) explicam que uma das causas do alto custo da produção de biodiesel derivado de microalgas vem da necessidade de fornecimento de grandes quantidades desses nutrientes e do alto consumo de água. Segundo Sialve et al. (2009), usando-se o nitrato como fonte de nitrogênio, seriam necessários entre 6-8 toneladas $\mathrm{ha}^{-1} \mathrm{ano}^{-1}$, quantidade entre 55111 maior que a necessária para culturas terrestres.

Águas residuais podem ser usadas como fontes dos nutrientes necessários ao cultivo, o que acabaria por reduzir os custos tanto do tratamento de água quanto da produção de biodiesel (CHEN et al., 2015). Segundo Gouveia et al. (2016), as microalgas liberam oxigênio durante a fotossíntese, o qual seria usado pelas bactérias no tratamento das águas residuais, consequentemente, diminuindo os custos de aeração do meio nesse processo. Outra vantagem descrita pelos autores é a fixação de metais pesados (selênio, crômio, chumbo), arsênio e compostos orgânicos nocivos (hidrocarbonetos), o que reduz a formação de lodo tóxico e a emissão de gases causadores do efeito estufa.

As águas residuais provêm de três fontes: esgoto doméstico, agrícola ou industrial. A maioria dos estudos combinando o tratamento de águas com a produção de biodiesel usa esgoto doméstico (CHEN et al., 2015). Kong et al. (2010) cultivaram Chlamydomonas reinhardtii em laboratório usando esse efluente e conseguiram uma produtividade máxima de biomassa de 2,9 $\mathrm{g} \mathrm{L}^{-1} \mathrm{dia}^{-1}$ e conteúdo lipídico de $25,25 \%$ da massa seca, para uma remoção de $55,8 \mathrm{mg} \mathrm{L}^{-1} \mathrm{~d}^{-1}$ de nitrogênio e $17,4 \mathrm{mg} \mathrm{L}^{-1} \mathrm{~d}^{-1}$ de fósforo. Li et al. (2011) provaram que Chlorella $s p$. são capazes de crescer nesse meio sem a 
necessidade de nenhum tratamento prévio. Os autores recuperaram microalgas com uma proporção de 11,04\% em massa seca de lipídios, alcançando uma produtividade de biodiesel de $0,12 \mathrm{~g} \mathrm{~L}^{-1}$ e recuperação da amônia, nitrogênio e fósforos totais presentes inicialmente de 93,9\%; 89, $1 \%$ e $80,9 \%$ respectivamente. Caporgno et al. (2015) compararam o desempenho de três diferentes espécies Chlorella kessleri, Chlorella vulgaris e Nannochloropsis oculata no cultivo em esgoto já pré-tratado. Nos resultados obtidos pelos autores as duas primeiras alcançaram altas concentrações de biomassa, $2,70 \pm 0,08 \mathrm{~g} \mathrm{~L}^{-1}$ e $2,91 \pm 0,02 \mathrm{~g} \mathrm{~L}^{-1}$ respectivamente, para uma recuperação de nitrogênio e fósforo de $99 \%$ e $98 \%$. Os resultados da terceira espécie foram bastante inferiores aos das outras duas, o que é atribuído ao fato da espécie ser natural de ambientes marinhos, sugerindo a necessidade de um processo de adaptação da espécie ao meio. Ainda segundo os autores, para todas as espécies a quantidade de lipídios acumulada foi insuficiente para a produção de biodiesel, mas pode ser melhorada através de mudanças nas condições de cultivo.

O esgoto agrícola tem maiores concentrações de nitrogênio e fósforo, quando comparado ao esgoto doméstico. Aproximadamente $50 \%$ do nitrogênio está na forma de amônia e a outra metade em compostos orgânicos (CAI et al., 2013). Franchino et al. (2013) cultivaram três espécies de microalgas (Scenedesmus obliquus, Neochloris oleoabundans e Chlorella vulgaris) usando resíduos de criação de gado e da produção de queijo previamente tratados em digestores anaeróbios. Segundo os autores, diluindo-se dez vezes o meio de cultivo foi possível recuperar quase $100 \%$ do nitrogênio e fósforo presentes inicialmente. A elevada turbidez provocada pela alta concentração de nutrientes gera a necessidade da diluição do meio de cultura, caso contrário a baixa penetração de luz prejudicaria o crescimento das microalgas (FENTON e HUALLACHÁIN, 2012). As razões de diluição de 1:1 e 1:2 acarretaram perda completa da biomassa, enquanto na diluição de $1: 5,87 \%$ da biomassa se perdeu (Franchino et al., 2013). Zhu et al. (2013) demonstraram em seu trabalho usando resíduos de tratamento de porcos que, não somente o crescimento da biomassa, mas a quantidade de lipídios acumulada também varia com a concentração inicial de nutrientes presente no meio de cultura. Assemany et al. (2016) cultivaram microalgas usando efluentes de uma unidade de processamento de carnes. O meio de cultura foi pré-tratado por dois métodos diferentes, flotação e lodo ativado. Comparando-se ambos, o acúmulo de lipídios foi próximo ( $7 \%$ e $6,1 \%$ respectivamente), porém para o primeiro caso a produtividade foi de $10 \mathrm{mg} \mathrm{L}^{-1} \mathrm{dia}^{-1}$, enquanto para o segundo foi de $3,4 \mathrm{mg} \mathrm{L}^{-1} \mathrm{dia}^{-1}$.

Os efluentes industriais são pouco estudados para a produção de biodiesel, uma vez que têm maior quantidade de metais e menor de nitrogênio e fósforo quando comparados aos demais. Por isso a maioria dos trabalhos foca no uso das microalgas para remoção dessas impurezas (CAI et al., 2013). Os resultados são promissores, pois reduzem os custos de produção e ainda está associado a método eficiente de tratamento de água (BATISTA et al., 2015). Segundo Chen et al. (2015), o que impede a adoção em larga escala é a baixa produtividade de biomassa e lipídeos. Alternativas para contornar esse problema têm sido buscadas no direcionamento das vias metabólicas das microalgas através do controle do fornecimento de nutrientes (CHEN et al., 2015).

Efluentes industriais gasosos podem ser usados como fonte de gás carbônico sem custos. O fornecimento desse gás é necessário porque a concentração dele presente na atmosfera não é suficiente para sustentar altas taxas de crescimento (SINGH, 2016). Praveenkumar et al. (2014) compararam o crescimento de microalgas em condições de cultivo autotrófica e mixotrófica usando efluentes gasosos de uma unidade de queima de carvão. Para Chlorella $s p$. sob condições de cultivo mixotróficas, o autor encontrou produtividade de biomassa de $561 \mathrm{mg} \mathrm{L}^{-1} \mathrm{dia}^{-1}$ e lipídios de $168 \mathrm{mg} \mathrm{L}^{-1} \mathrm{dia}^{-1}$. Ambos os resultados são superiores aos obtidos pelas culturas autotróficas, porque na primeira condição as microalgas conseguem manter o crescimento mesmo na ausência de luz, mas, para isso, é necessário o fornecimento de carbono orgânico (PRAVEENKUMAR et al., 2014). Cheah et al. (2015) ressaltam que nem todas as espécies se beneficiam da elevada concentração de $\mathrm{CO}_{2}$ presente nos efluentes gasosos industriais (entre 6-15\% em volume, enquanto o no ar atmosférico varia entre 0,03 e $0,06 \%$ ). Além disso, o autor aponta outros fatores que inibem o uso desses efluentes, como a alta temperatura, a presença de outros gases tóxicos que podem inibir o crescimento ou alterar o $\mathrm{pH}$ do meio de cultivo, e a presença de metais pesados, que, em determinadas concentrações, também podem inibir o crescimento.

\subsection{Forma de Cultivo}

Do ponto de visto tecnológico, existem duas formas de cultivo: sistemas abertos (raceway ponds, lagos, etc.) e sistemas fechados (tubular, fermentador, piramidal, reator em placa, cônico, etc.) (DASGUPTA et al., 2010).

Os sistemas de cultivos abertos podem ser construídos de uma enorme variedade de formas e tamanhos. Raceway (tipo pista de corrida) é o sistema mais difundido atualmente e o mais antigo, tendo sido empregado desde 1950 (Figura 1A) (PEQUENO et al., 2012). De acordo com Huang et al. (2015), esses sistemas consistem em tanques abertos ao ar livre, geralmente com $20-50 \mathrm{~cm}$ de profundidade, agitação e circulação do meio de cultivo visando estabilizar o crescimento e a produtividade das microalgas. Os autores explicam que agitação contínua impede que células se depositem no fundo e garantem melhor acesso aos nutrientes e a luz solar. As microalgas e os nutrientes são introduzidas na frente de uma pá mecânica de agitação, e movidas até o final do tanque onde são coletadas. A fonte de carbono provém do ar, podendo ser instalados aeradores de modo a aumentar a absorção de $\mathrm{CO}_{2}$ (BRENNAN \& OWENDE, 2010, DASGUPTA et al., 2010). Segundo Franco et al. (2013), os sistemas abertos têm menores custos, tanto de construção quanto de operação. Os autores explicam que os custos de construção são menores porque diversos materiais podem ser usados na criação das paredes e dos fundos dos tanques, geralmente concreto, mas podem ser usados também tijolos, areia, plástico, etc. 


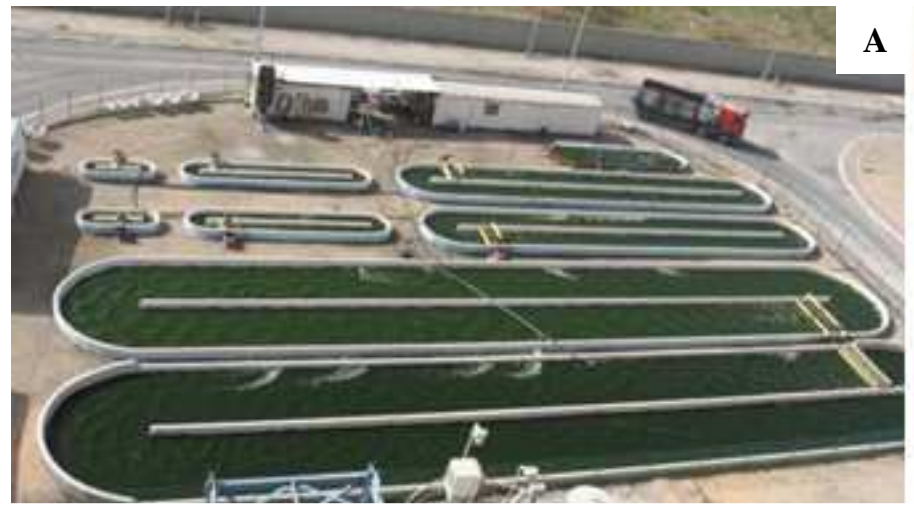

Figura 1. Sistemas de cultivo de microalgas. O sistema aberto (A), e o sistema fechado (B). Fonte: ADENIYI; AZIMOV;

BURLUKA (2018).

A manutenção do sistema aberto é mais simples em comparação aos sistemas fechados, pela facilidade de acesso ao tanque para a limpeza do biofilme que se acumula sobre as superfícies após o cultivo (FRANCO et al., 2013). A desvantagem desse sistema é a dificuldade de controle das variáveis de cultivo (temperatura, iluminação, salinidade, $\mathrm{pH}$, etc) que estão muito sujeitas a influência das condições ambientais (CHISTI et al., 2007). Além disso, Kumar et al. (2015) citam que ocorrem grandes perdas de água por evaporação, a eficiência do uso do $\mathrm{CO}_{2}$ é baixa e existem grandes riscos de contaminação por espécies indesejáveis, que diminuem o rendimento e são de difícil erradicação depois de se estabelecerem no interior dos tanques. Esses fatores tornam a produtividade dos sistemas abertos muito inferior à dos sistemas fechados (RAWAT et al., 2013). Segundo Brennan e Owende (2010), a produtividade volumétrica alcançada nos sistemas abertos varia entre $0.12-0.48 \mathrm{~g} \mathrm{~L}^{-1} \mathrm{~d}^{-1}$, ao passo que nos fechados varia entre $0.2-3.8 \mathrm{~g} \mathrm{~L}^{-1} \mathrm{~d}^{-1}$. A Tabela 3 exibe um comparativo entre os sistemas de cultivo aberto e fechado.

O cultivo fechado é feito em foto biorreatores, que são uma série de tubos transparentes, geralmente de vidro ou plástico, que funcionam como coletores de luz solar, onde são controlados: a temperatura, o $\mathrm{pH}$ e o fornecimento dos nutrientes e de $\mathrm{CO}_{2}$ (Figura 1B) (CHISTI, 2007). Esses foto-biorreatores podem ser encontrados em diferentes formas: reator tubular vertical; reator tubular horizontal; reator tubular helicoidal; reator em placas, que se caracterizam por ter uma área aberta para trocas gasosas; reator tipo fermentador, no qual a iluminação é fornecida internamente (DASGUPTA et al., 2010).

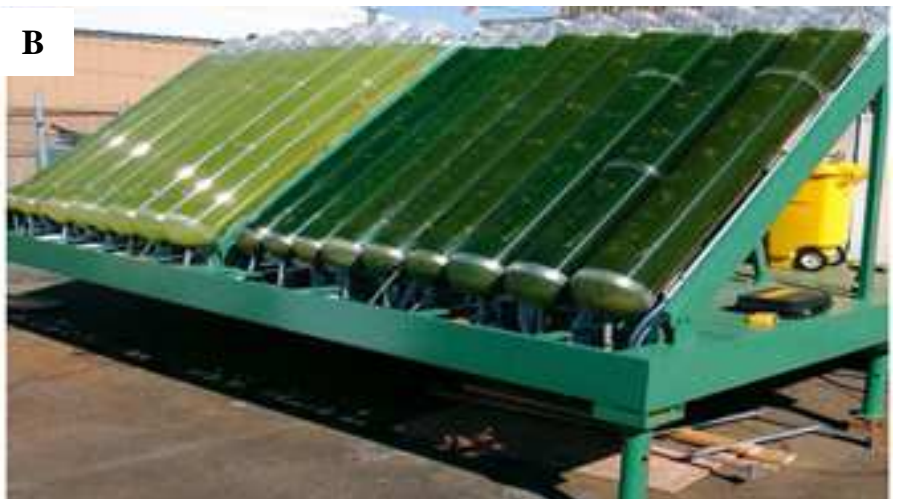

As condições de cultivo controladas, a melhor distribuição da luz no interior do reator e a diminuição dos riscos de contaminação, tanto por outras algas quanto por insetos, tornam a produtividade volumétrica dos foto-biorreatores oito vezes superior, e a concentração celular 16 vezes superior ao dos sistemas abertos (PEQUENO et al., 2012). Entretanto, os custos de construção, operacionais e de manutenção são maiores, além de outros problemas possíveis como o superaquecimento (acompanhado da necessidade de sistema de resfriamento), entupimento, e acúmulo de oxigênio no interior do reator (Brenna e Owende, 2010).

Chinnasamy et al. (2011) estudaram em laboratório o cultivo de microalgas em três diferentes sistemas de cultivo fechados (lagoa raceway, tanques verticais e polybags) usando efluente de indústria de tapetes sem nenhum tratamento. Os resultados demonstram que os reatores de Polybags são mais vantajosos, com produtividade por área de $21,1 \mathrm{~g} \mathrm{~m}^{-2} \mathrm{dia}^{-1}$ enquanto os sistemas de lagoa raceway e tanques verticais apresentaram produtividade de 5,9 e $8,1 \quad \mathrm{~g} \mathrm{~m}^{-2} \mathrm{dia}^{-1}$ respectivamente. Polybags com capacidade de $30 \mathrm{~L}$ permitiram produtividade de 77 ton ha $^{-1}$ ano $^{-1}$. A biomassa produzida no experimento era rica em proteínas $(53,8 \%)$ e pobre em carboidratos $(15,7 \%)$ e lipídios $(5,3 \%)$.

Após o cultivo da microalga, a produção de biodiesel segue para a etapa de processamento da biomassa, que passa pela colheita, secagem e extração do óleo produzido.

Tabela 3 - Comparativo dos sistemas de cultivo abertos e fechados.

$\begin{array}{lll}\text { Parâmetro } & \text { Sistemas abertos } & \text { Sistemas Fechados } \\ \text { Espaço necessário } & \text { Grande } & \text { Pequeno } \\ \text { Risco de Contaminação } & \text { Alto } & \text { Médio a baixo } \\ \text { Perdas de Água } & \text { Alto } & \text { Baixo } \\ \text { Perdas de CO2 } & \text { Alto } & \text { Quase nenhuma } \\ \text { Concentração de O2 } & \text { Baixa } & \text { Deve ser removido continuamente } \\ \text { Controle do processo } & \text { Limitado } & \text { Possível } \\ \text { Desgaste dos materias de construção } & \text { Baixo } & \text { Alto } \\ \text { Dependência das condições ambientais } & \text { Alto } & \text { Baixo, por ser fechado. } \\ \text { Temperatura } & \text { Variável } & \text { Necessita resfriamento } \\ \text { Custos da colheita } & \text { Alto } & \text { Médio } \\ \text { Manutenção } & \text { Fácil } & \text { Difícil } \\ \text { Custos de construção } & \text { Médio } & \text { Alto } \\ \text { Concentrações de biomassa na colheita } & \text { Baixo } & \text { Alto }\end{array}$

Fonte: Pereira, 2012. 


\section{COLHEITA}

A colheita é a etapa na qual a biomassa de microalgas é separada do grande volume de água. A técnica ideal de colheita deve ser capaz de produzir biomassa de alta concentração, com baixos custos operacionais, energéticos e de manutenção, porém, até o momento não foi encontrado uma técnica que atenda a esses requisitos (BARROS et al., 2015). Segundo Grima et al. (2003) a dificuldade do processo reside na alta diluição dos meios de cultura $(0,5-2 \mathrm{~g} / \mathrm{L}$ em tanques abertos e $5-10 \mathrm{~g} / \mathrm{L}$ em fotobiorreatores), que o torna responsável por entre 20 a $30 \%$ dos custos totais de produção. Para um determinado processo, Pragya et al. (2013) explicam que a escolha do método mais adequado depende de vários fatores, como a concentração do meio de cultivo, o tamanho das microalgas, a vazão do meio a ser separado e a especificação desejada para o produto final. Atualmente, os mais comuns são sedimentação por gravidade, centrifugação, filtração, ultra filtração, flotação, floculação, ou uma combinação entre eles (BARROS et al., 2015).

A centrifugação é um método de colheita que pode ser usado para a maioria das espécies de microalgas (RAWAT et al., 2011). Segundo Barros et al. (2015) esse é o processo mais rápido de colheita, porém o mais caro, devido ao alto consumo energético que o torna aplicável somente na produção de produtos de alto valor. De acordo com Pragya et al. (2013), ensaios em laboratório foram feitos com efluentes de lagos e conseguiram recuperar cerca de $80-90 \%$ das microalgas dentro de 2 a 5 minutos. Aumento na concentração em até 150 vezes e eficiências de colheita superiores a 95\% são tecnicamente viáveis (GERARDO et al., 2015).

A sedimentação por gravidade é o método mais simples e barato de colheita, mas é lento e pouco eficiente, sendo aplicável somente para microalgas grandes, uma vez que as de baixa densidade permanecem em suspensão e acabam não sendo separadas no processo (UDUMAN et al., 2010). De acordo com Show et al. (2014) a colheita de microalgas por esse método é feita em separadores de lamela, para uma recuperação de $6 \%$ dos sólidos totais em suspensão, e tanques de sedimentação, onde $3 \%$ de sólidos totais em suspensão são recuperados. Esses resultados mostram a baixa eficiência do processo e a necessidade da aplicação de outros métodos de recuperação da biomassa após a sedimentação por gravidade.

A floculação é um processo que visa agregar partículas menores de forma a facilitar a sua sedimentação (GERARDO et al., 2015). Existem várias formas de se obter a floculação. Segundo Vandamme et al. (2013), a auto floculação ocorre pela precipitação de sais com algas em $\mathrm{pH}$ elevado. De acordo com o autor o aumento do $\mathrm{pH}$ é provocado pelo consumo de $\mathrm{CO}_{2}$ na fotossíntese. Assim, o cultivo sob luz solar com fornecimento restrito de $\mathrm{CO}_{2}$ auxilia a auto floculação de células.

Tabela 4 - Comparativo entre os métodos de colheita de microalgas mais usados.

\begin{tabular}{|c|c|c|c|}
\hline $\begin{array}{l}\text { Processo de } \\
\text { Colheita }\end{array}$ & Rendimento & Gasto Energético & Desvantagens \\
\hline Floculação & $\begin{array}{c}\text { Acima de } 95 \% \text { de remoção das } \\
\text { microalgas. }\end{array}$ & Baixa para fraca agitação & $\begin{array}{c}\text { Custo dos floculantes e risco } \\
\text { de contaminação. }\end{array}$ \\
\hline Centrifugação & $\begin{array}{l}95 \% \text { de colheita de células e } 22 \% \text { de } \\
\text { sólidos suspensos totais. }\end{array}$ & Alta, $8 \mathrm{KWh} / \mathrm{m} 3$ & Consumo de energia. \\
\hline Sedimentação & 0,5-1,5\% de sólidos suspensos totais. & Baixa, $0,1 \mathrm{kWh} / \mathrm{m}^{3}$ & Processo lento. \\
\hline Filtração & 1-6\% de sólidos suspensos totais. & Baixa, $0,4 \mathrm{kWh} / \mathrm{m}^{3}$ & $\begin{array}{c}\text { Filtros precisam ser trocados } \\
\text { periodicamente. }\end{array}$ \\
\hline Filtração (pressão) & $5-27 \%$ de sólidos suspensos totais. & Média, $0,88 \mathrm{kWh} / \mathrm{m}^{3}$ & $\begin{array}{c}\text { Filtros precisam ser trocados } \\
\text { periodicamente. }\end{array}$ \\
\hline $\begin{array}{l}\text { Floculação - } \\
\text { Flotação }\end{array}$ & $\begin{array}{l}90 \% \text { de remoção de microalga e } 1-6 \% \\
\text { de sólidos suspensos totais. }\end{array}$ & \multicolumn{2}{|c|}{ Alta, 10 a $20 \mathrm{kWh} / \mathrm{m}^{3}$} \\
\hline Eletrocoagulação & $\begin{array}{l}\text { Acima de } 95 \% \text { de remoção das } \\
\text { microalgas. }\end{array}$ & $\begin{array}{l}\text { Média-Alta, } 0,8 \text { a } 1,5 \\
\qquad \mathrm{kWh} / \mathrm{m}^{3}\end{array}$ & $\begin{array}{l}\text { Eletrodos precisam ser } \\
\text { trocados periodicamente. }\end{array}$ \\
\hline Eletroflotaçao & 3-5\% de sólidos suspensos totais. & Elevada & $\begin{array}{l}\text { Eletrodos precisam ser } \\
\text { trocados periodicamente. }\end{array}$ \\
\hline Eletrofloculação & $\begin{array}{c}\text { Acima de } 90 \% \text { de remoção das } \\
\text { microalgas. }\end{array}$ & Baixa-média, 0,33 kWh/m³ & $\begin{array}{l}\text { Eletrodos precisam ser } \\
\text { trocados periodicamente. }\end{array}$ \\
\hline
\end{tabular}

Fonte: Santos, 2013.

Alternativamente, é possível empregar produtos químicos (inorgânicos ou orgânicos) com capacidade coagulante. Eles são responsáveis por neutralizar as cargas negativas presentes na superfície das microalgas, o que diminui a repulsão entre elas e permite a agregação das partículas (GERALDO et al., 2015). Os inorgânicos mais usados são à base de ferro e alumínio, por serem baratos e acessíveis (CHATSUNGNOEN e CHISTI, 2016). Usando sulfato de alumínio, Chatsungnoen e Chisti (2016) conseguiram separar dois terços da água presente na suspensão contendo as microalgas. As desvantagens no uso dos coagulantes inorgânicos são a necessidade de grandes concentrações da substância, elevada sensibilidade ao $\mathrm{pH}$, especificidade para determinadas espécies de algas e a contaminação do produto final com os metais adicionados (UDUMAN et al., 2010). Os floculantes orgânicos usados são polímeros de alto peso 
molecular, sendo os catiônicos os mais eficazes (VANDAMME et al., 2013).

A filtração convencional opera sob pressão ou sucção, e auxiliares de filtração como celulose e terra diatomácea podem ser usados para aumentar a eficiência (GRIMA et al., 2003) De acordo com Brennan e Owende (2010), esse método é indicado para algas grandes, maiores que $70 \mathrm{~mm}$, não podendo ser usado para algas de dimensões inferiores a $30 \mathrm{~mm}$. Segundo os autores, para algas menores que $30 \mathrm{~mm}$, é possível usar a micro e ultra filtração por membranas, que também são indicadas para células frágeis. Mohn (1980) conseguiu, por meio da filtração comum, concentrar em até 245 vezes suspensões contendo microalgas, com formação de tortas contendo $27 \%$ de sólidos. Já com a filtração por membranas é possível recuperar $100 \%$ da biomassa (GERARDO et al., 2015), porém ainda não aplicável em larga escala devido ao alto consumo energético e a necessidade de substituições frequentes das membranas (RAWAT et al., 2013).

Flotação é baseada no aprisionamento das células de microalgas usando micro-bolhas de ar dispersos no cultivo. As bolhas se aderem às partículas sólidas e as levam para a superfície do líquido. Para a geração de bolhas, pode ser usado ar disperso, ar dissolvido, eletrólise ou ozônio (BARROS et al., 2015). Gerardo et al. (2015) explicam que a flotação com ar dissolvido usa um fluxo de água que é pré-saturada com ar para produzir bolhas de 10 a $100 \mu \mathrm{m}$ que o torna mais eficiente e o mais amplamente utilizado. Essa flotação tem sido geralmente usada combinada com a floculação química na separação de microalgas, e resultados melhores têm sido alcançados em relação aos obtidos com a sedimentação (UDUMAN et al., 2010). De acordo com Laamanen et al. (2016), na flotação com ar disperso as bolhas são geradas através de um agitador mecânico. Segundo os autores são geradas bolhas entre 700$1500 \mu \mathrm{m}$, o que limita o seu uso, uma vez que as bolhas menores são mais eficientes em se aderir às partículas sólidas. $\mathrm{Na}$ flotação com gás ozônio, são geradas bolhas carregadas eletricamente, que acabam por interagir com as células das microalgas que naturalmente contem cargas negativas em sua superfície (BARROS et al., 2015).

$\mathrm{Na}$ floculação eletrolítica, as microalgas são atraídas para o ânodo e lá suas cargas superficiais são neutralizadas, dessa forma, possibilitando a agregação das partículas. Já a eletrocoagulação usa eletrodos de sacrifício, que por meio de oxidação eletrolítica, formam o coagulante que desestabiliza as partículas em suspensão que acabam por formar flocos (AZARIAN et al., 2007). A Tabela 4 mostra um comparativo entre os mais aplicados métodos de colheita de microalgas.

\section{SECAGEM E EXTRAÇÃO DO ÓLEO}

Após a colheita, as microalgas passam pelo processo de secagem. De acordo com Lam e Lee (2012), a ausência de umidade é importante para os processos seguintes de extração do óleo e transesterificação. A secagem ao sol é o método mais simples e barato, mas é lento, exigindo grandes áreas de secagem e tornando o processo sujeito a perdas de material (LAM e LEE, 2012). Este processo de secagem é inviável em países de clima temperado. Segundo Sander e Murthy (2010), as baixas temperaturas desses países implicam no gasto de combustíveis para possibilitar a secagem, entretanto a energia gasta nesse processo pode superar àquela produzida como biodiesel em si, o que presumivelmente inviabiliza sua produção comercial.

Nesses casos, é necessário o desenvolvimento de melhores tecnologias de secagem, ou o desenvolvimento de processos de extração e transesterificação que sejam pouco sensíveis ou insensíveis a presença de água (LAM e LEE, 2012). Outros dois métodos de secagem são a liofilização e a secagem em prateleiras, porém eles são muito caros e não aplicáveis em larga escala, sendo somente viáveis para produtos de alto valor agregado (GRIMA et al, 2003).

A extração do óleo das microalgas pode ser feita por meio de processos físicos, químicos, da combinação dos dois, com enzimas ou com fluido supercrítico. Segundo Fajardo et al. (2007), o método usado deve ser o mais seletivo e especifico possível, a fim de se extrair somente a fração desejada. Os autores explicam que as microalgas produzem diversos tipos de lipídeos, como fosfolipídeos, glicolipídeos, monoacilgliceróis, diacilgliceróis, triacilgliceróis, além de outros componentes não lipídicos, como poilissacarídeos, proteínas e pigmentos. Porém, dentre todos eles, somente os triacilgliceróis podem ser convertidos facilmente em biodisel pelo processo de transesterificação que é o mais usado atualmente em escala comercial. Outra possibilidade seria a extração não seletiva e a destinação dessas outras substâncias para a indústria alimentícia e farmacêutica, o que acabaria por reduzir os custos de produção de biodiesel (FRANCO et al., 2013).

Os processos mecânicos de extração rompem a parede celular das células para provocar a liberação do óleo (KIM et al., 2013). De acordo com Rashid et al. (2014), são vários os métodos mecânicos usados, cada um deles com vantagens e desvantagens. A eficiência alcançada está ligada à espécie de microalga em questão e melhores resultados geralmente são obtidos quando os métodos mecânicos são combinados a processos químicos (RASHID et al., 2014).

Greenwell et al. (2010) explicam que a prensagem mecânica usa altas pressões para romper a parede das células. De acordo com os autores, a vantagem desse método, comum aos demais processos mecânicos, está na redução dos riscos de contaminação da biomassa das microalgas e na conservação das características químicas das substâncias presentes no interior das mesmas. Outra técnica mecânica usa ondas sonoras de alta frequência que geram microbolhas, calor e ondas de choque que podem romper as paredes celulares. Esse método não pode ser usado para todas as espécies de microalgas, além de consumir muita energia, o que inviabiliza sua aplicação em larga escala (RASHID et al., 2014). Já o método da pressão osmótica provoca o rompimento celular pela redução repentina da pressão osmótica. O processo é barato, mas não é capaz de romper todas as células (FRANCO et al., 2013).

Os processos químicos utilizam solventes orgânicos. Os mais usados são o clorofórmio, hexano, benzeno, ácidos, etanol e álcalis (Rashid et al., 2014). Segundo os autores, para o processo ser eficiente é necessário que o solvente penetre completamente na biomassa e entre em contato com os lipídeos. Além disso, o solvente deve ser insolúvel em água, deve dissolver a substância que se quer extrair, ter baixo ponto de ebulição e densidade muito diferente da água, de maneira a facilitar a separação do solvente e do óleo após o processo de extração. A extração com biomassa seca é mais eficiente, porque a presença de umidade reduz a interação entre as moléculas do solvente e a biomassa (HALIM et al., 2012). Os 
elevados custos de secagem tornam inviáveis economicamente a extração com a biomassa seca. Segundo Kim et al. (2013), apesar da alta eficiência alcançada com a extração química, ela tem algumas desvantagens, como a necessidade de alta quantidade de solvente para baixas concentrações de biomassa, altos custos relacionados a recuperação do solvente usado e a geração de grande quantidade de resíduos. Além disso, o solvente usado pode alterar a composição química das substâncias extraídas.

Tabela 5 - Comparativo entre os métodos de extração de óleo de microalgas.

\section{Método de Extração \\ Vantagens}

Prensagem

Extração com Solvente

Extração com Fluido Supercrítico

Ultrassom

Enzimático

Fonte: Mubarak et al., 2015.
Facilidade de aplicação, não usa produtos químicos.

Solventes usados são relativamente baratos.

Não é tóxico nem inflamável, não deixa resíduos de solvente no extrato.

Extração rápida reduz a quantidade de solvente usada, melhor ruptura da parede celular.

Seletivo, não altera quimicamente os lipídeos.

\section{Desvantagens}

Processo lento que requer uma grande quantidade de matéria prima

Maioria dos solventes orgânicos são inflamáveis e/ou tóxicos, são necessárias grandes quantidades de solvente e a recuperação é cara.

Alto custo

Alto Custo

Custo elevado das enzimas

Tabela 6 - Avanços recentes no processo produtivo de biodiesel a partir de microalgas.

\section{Extração/Conversão} Cultivo Colheita

Solventes (etanol + hexano)

Diversos solventes de fontes renováveis

Transesterificação Básica

$\mathrm{CO}_{2}$ supercrítico

Solventes eutéticos profundos +

Micro-ondas+dimetilcarbonato $+\mathrm{CO}_{2}$ supercrítico

Micro-ondas+ $\mathrm{CO}_{2}$ supercrítico +

mistura de solventes azeotrópicos

Solventes Comutáveis

Transesterificação com catalizador enzimático

Micro-ondas

Ultrassom

Pulso elétrico

Mistura (etanol + hexano)

Hidrólize enzimática

$\mathrm{CO}_{2}$ supercrítico

Catalizador Enzimático

Extração e conversão simultâneas

Solventes comutáveis

Catálise enzimática

Extração e conversão simultâneas

Metanol supercrítico na presença de

$\mathrm{CaO} / \mathrm{TiO}_{2}$

Ultrassom

Solventes Bifásicos

Transesterificação com catalizador

heterogêneo ( $\mathrm{Mn}-\mathrm{ZnO}$ encapsulado em

polietileno glicol)

Conversão direta via saponificaçãoesterificação
Foto-biorreatores

Foto-biorreatores

Sistema

aberto/foto-

biorreatores

Foto-biorreatores

Centrifugação

Tommasi et al., (2017)

\section{Flotação + Centrifugação}

Escorsim et al., (2018)

Wan Mahmood,

Theodoropoulos, \&

Gonzalez-Miquel, (2017)

Lorenzen et al., (2017)

Patil, Dandamudi, Wang,

Deng, \& Deng, (2018)

Al-Ameri \& Al-Zuhair, (2019)

Sistema aberto
(pista de corrida) $\quad$ Centrifugação

Ansari, Gupta, Nasr, Rawat, \& Bux, (2018)

Silve et al., (2018)

Sistema aberto/

Foto-biorreatores

Decantação

Foto- biorreatores

Zhang et al., (2018)

Foto- biorreatores

Shomal, Hisham, Mlhem,

Hassan, \& Al-Zuhair, (2019)

Sistema aberto
(pista de corrida)

Centrifugação

Al-Ameri \& Al-Zuhair, (2019)

Foto- biorreatores Centrifugação

Aghilinategh, Barati, \& Hamadanian, (2019)

Vinoth Arul Raj et al., (2019)

Centrifugação

Fang, Yeh, \& Liu, (2018) 
Fluídos supercríticos têm alcançado excelentes resultados na extração de óleo (VIGUERA et al., 2016). O mais usado é o $\mathrm{CO}_{2}$, devido à temperatura $\left(31,1^{\circ} \mathrm{C}\right)$ e pressão críticas (72,9 atm) relativamente baixas (CONNEY et al., 2009). Segundo Viguera et al. (2016) a vantagem dessa técnica é a alta seletividade na extração. De acordo com os autores, a eficiência depende da temperatura, da pressão, do tempo de extração e da vazão do fluido. Devido aos altos custos, atualmente só é aplicável para a extração de produtos de maior valor agregado (VIGUERA et al., 2016).

O uso de enzimas para a extração do óleo se mostra promissora. A eficiência do processo é alta, e tem a vantagem sobre os processos químicos de não alterar quimicamente os lipídeos. As classes de enzimas mais usadas são: neutrase, celulase e pectinase. A desvantagem desse método é o alto custo das enzimas (RASHID, 2014). A Tabela 5 resume os métodos de extração do óleo das microalgas.

\section{PERSPECTIVAS FUTURAS}

A produção de biodiesel a partir de microalgas é viável tecnicamente, como descrito anteriormente, entretanto ainda existem obstáculos acerca da viabilidade econômica. Os custos da produção são altos, e superam os custos do diesel do petróleo e até mesmo de biodiesel provindo de outras matérias primas (GUPTA et al., 2016).

Segundo Azeredo (2012), as etapas de cultivo e recuperação da biomassa são os principais responsáveis pelo custo de capital associado a esses processos. Os custos operacionais em contrapartida estão associados ao consumo de nutrientes e principalmente $\mathrm{CO}_{2}$. $\mathrm{O}$ autor ainda ressalta que pesquisa e desenvolvimento podem ser capazes de reduzir os custos de produção tornando o processo competitivo em relação às culturas tradicionais.

Avanços na etapa de colheita das microalgas são necessários, e as pesquisas caminham na direção do desenvolvimento de floculantes naturais. Estes já são usados com sucesso nos processos de tratamento de água, porém na produção de biodiesel ainda carecem de avanços no sentido de tornar o processo mais rápido (VANDAMME et al.,2013). As pesquisas também buscam o aperfeiçoamento do processo de extração, com o objetivo de recuperar o óleo na presença de umidade, de modo a tornar desnecessária a etapa de secagem, que é muito custosa energeticamente (CHEN et al., 2015). Avanços recentes nas diversas etapas do processo produtivo de biodiesel a partir de microalgas são sumarizados na Tabela 6 . Melhorias alternativas no processo de produção são: alterações genéticas e identificação de novas espécies de microalgas; desenvolvimento de foto biorreatores; além do melhor aproveitamento da biomassa (RASHID et al., 2014).

Engenharia genética e metabólica terá provavelmente o maior impacto na viabilidade econômica do biodiesel de microalgas, porém pouco avanço foi alcançado nessa área. Os objetivos nesse desenvolvimento são: microalgas com maior eficiência fotossintética, de modo a aumentar a produção de biomassa durante os períodos em que estão expostas à luz; maior taxa de crescimento de biomassa; aumento da quantidade de óleo no interior das microalgas, aumento da tolerância a altas temperaturas, possibilitando menores gastos com o resfriamento dos foto biorreatores; aumento da resistência a oxidação provocada pela luz; eliminação da foto inibição, que provoca a parada da atividade fotossintética quando o luminosidade atinge certo nível, de forma que o crescimento sempre aumente em resposta ao incremento da luminosidade (RASHID et al., 2014).

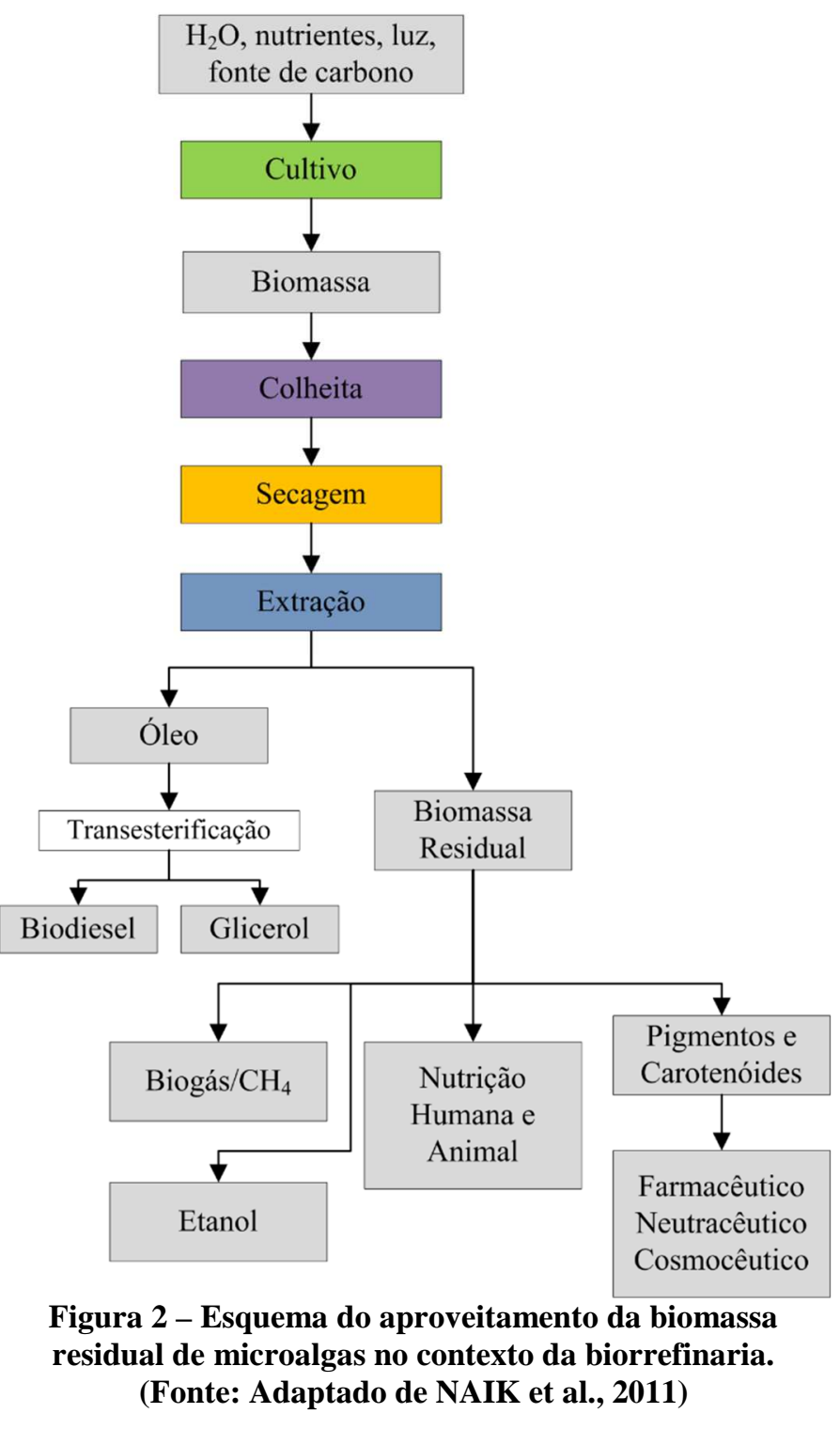

O melhor aproveitamento da biomassa gerada na produção do óleo pelas microalgas é uma promissora alternativa para alcançar a viabilidade econômica. Esse aproveitamento está associado ao conceito de biorrefinaria, ou seja, um complexo industrial que integra os processos de produção de biodiesel ao aproveitamento da biomassa residual para produção de produtos com alto valor (RIZWAN et al., 2015). Uma vez que as microalgas contêm quantidades significativas de proteínas, carboidratos e outros nutrientes, ela pode ser usada na produção de antibióticos (BURJA et al., 2001), clorofila (YAGUI et al., 2004), enzimas (RAPOSO et al., 2013), ésteres (YEN et al., 2013), hidrocarbonetos (DU et al., 2013), vitaminas (MARKOU e NERANTZIS, 2013), lipídeos carotenóides (RODRIGUES et al., 2014), lipídeos (POHNDORF et al., 2016), proteínas (SUGANYA et al., 2016), e empregadas na produção de ração animal (SUGANYA et al., 2016). Ainda podem ser empregados na produção de energia através digestão anaeróbia e produção de metano, que por sua vez pode ser usado na própria planta ou seu excedente vendido, prática já observada em outras biorrefinarias (MILANO et al., 2015). A Figura 2 exibe o esquema do 
processo produtivo integrado, no conceito de biorrefinaria, empregando microalgas como matéria-prima.

\section{CONCLUSÃO}

As microalgas apresentam características muito particulares que as tornam, atualmente, uma das fontes mais promissora de biomassa para a produção de biodiesel no mundo. Trata-se da matéria-prima mais promissora para substituição completa dos combustíveis fósseis por renováveis, uma vez que não compromete áreas plantadas para a produção de alimentos. Os aspectos fisiológicos e bioquímicos permitem alta produtividade e acúmulo de lipídios, além de apresentarem grande potencial para melhoramento. Os aspectos sociais de produção intensiva e tratamento de águas residuais corroboram suas vantagens. Entretanto, em relação ao processo produtivo, observou-se alguns entraves que dificultam a aplicação desta tecnologia em grande escala, inviabilizando-a economicamente. A alta diluição dos meios de cultivo, a necessidade de processos de secagem eficientes, e consequentemente $o$ alto gasto energético são alguns dos fatores que devem centrar as novas pesquisas. $\mathrm{O}$ avanço das tecnologias disponíveis permitirá o amplo emprego das microalgas para a produção de biodiesel como fonte renovável de energia no futuro.

\section{R E F E R Ê N C IAS}

AGÊNCIA NACIONAL DO PETRÓLEO, GÁS NATURAL E BIOCOMBUSTÍVEIS (ANP). Disponível em: <http://www.anp.gov.br/>. Acesso em: 29 jan. 2019.

AGHILINATEGH, M.; BARATI, M.; HAMADANIAN, M. Supercritical methanol for one put biodiesel production from chlorella vulgaris microalgae in the presence of $\mathrm{CaO} / \mathrm{TiO} 2$ nano-photocatalyst and subcritical water. Biomass and Bioenergy, v. 123, p. 34-40, fev. 2019.

AL-AMERI, M.; AL-ZUHAIR, S. Using switchable solvents for enhanced, simultaneous microalgae oil extractionreaction for biodiesel production. Biochemical Engineering Journal, v. 141, p. 217-224, out. 2019.

AMARO, H. M.; GUEDES, A. C.; MALCATA, F. X. Advances and perspectives in using microalgae to produce biodiesel. Applied Energy, v. 88, n. 10, p. 3402-3410, out. 2011.

ANGEL-YAGUI, C. DE O., DANESI, E. D. G., DE CARVALHO, J. C. M., \& SATO, S. Chlorophyll production from Spirulina platensis: cultivation with urea addition by fed-batch process. Bioresource Technology, v. 92, n. 2, p. 133-141, abr. 2004.

ANSARI, F. A. et al. Evaluation of various cell drying and disruption techniques for sustainable metabolite extractions from microalgae grown in wastewater: A multivariate approach. Journal of Cleaner Production, v. 182, p. 634-643, maio 2018.

ANUAR, M. R.; ABDULLAH, A. Z. Challenges in biodiesel industry with regards to feedstock, environmental, social and sustainability issues: A critical review. Renewable and Sustainable Energy Reviews, v. 58, p. 208-223, maio 2016.

ASSEMANY, P. P., CALIJURI, M. L., TANGO, M. D., \& COUTO, E. A. Energy potential of algal biomass cultivated in a photobioreactor using effluent from a meat processing plant. Algal Research, v. 17, p. 53-60, 1 jul. 2016.

AZARIAN, G., MESDAGHINIA, A., VAEZI, F., NABIZADEH, R., \& NEMATOLLAHI, D. Algae Removal by Electro-coagulation Process, Application for Treatment of the Effluent from an Industrial Wastewater Treatment Plant. Iranian J Publ Health, v. 36, n. 4, p. 57-64, 2007.

AZEREDO, V. B. S. Produção de biodiesel a partir do cultivo de microalgas: Estimativa de custos e perspectiva de custos para o Brasil. Dissertação (Mestrado em Planejamento Energético) Universidade Federal do Rio de Janeiro, RJ, 2012.

BANERJEE A1, SHARMA R, CHISTI Y, BANERJEE UC.. Botryococcus braunii: A renewable source of hydrocarbons and other chemicals. Critical Reviews Biotechnology, p.22, 245, 2002.

BARROS, A. I., GONÇALVES, A. L., SIMÕES, M., \& PIRES, J. C. M. Harvesting techniques applied to microalgae: A review. Renewable and Sustainable Energy Reviews, v. 41, p. 1489-1500, jan. 2015.

BATISTA, A. P., AMBROSANO, L., GRAÇA, S., SOUSA, C., MARQUES, P. A. S. S., RIBEIRO, B., GOUVEIA, L. Combining urban wastewater treatment with biohydrogen production - An integrated microalgaebased approach. Bioresource Technology, v. 184, p. 230235, maio 2015.

BORUGADDA, V. B.; GOUD, V. V. Biodiesel production from renewable feedstocks: Status and opportunities. Renewable and Sustainable Energy Reviews, v. 16, n. 7, p. 4763-4784, 1 set. 2012.

BRENNAN, L.; OWENDE, P. Biofuels from microalgae-A review of technologies for production, processing, and extractions of biofuels and co-products. Renewable and Sustainable Energy Reviews, v. 14, n. 2, p. 557-577, 1 fev. 2010.

BURJA, A. M., BANAIGS, B., ABOU-MANSOUR, E., GRANT BURGESS, J., \& WRIGHT, P. C. Marine cyanobacteria - a prolific source of natural products. Tetrahedron, v. 57, n. 46, p. 9347-9377, 12 nov. 2001.

CAI, T.; PARK, S. Y.; LI, Y. Nutrient recovery from wastewater streams by microalgae: Status and prospects. Renewable and Sustainable Energy Reviews, v. 19, p. 360-369, 1 mar. 2013.

CAPORGNO, M. P., TALEB, A., OLKIEWICZ, M., FONT, J., PRUVOST, J., LEGRAND, J., \& BENGOA, C. Microalgae cultivation in urban wastewater: Nutrient removal and biomass production for biodiesel and methane. Algal Research, v. 10, p. 232-239, 1 jul. 2015.

CHATSUNGNOEN, T.; CHISTI, Y. Continuous flocculationsedimentation for harvesting Nannochloropsis salina biomass. Journal of Biotechnology, v. 222, p. 94-103, mar. 2016.

CHEAH, W. Y., SHOW, P. L., LING, T. C., \& JUAN, J. C. Biosequestration of atmospheric $\mathrm{CO} 2$ and flue gascontaining $\mathrm{CO} 2$ by microalgae. Bioresource Technology, v. 184 , p. 190-201, 1 maio 2015.

CHEN, C.-L., HUANG, C.-C., HO, K.-C., HSIAO, P.-X., WU, M.-S., CHANG, J.-S. Biodiesel production from wet microalgae feedstock using sequential wet extraction/transesterification and direct transesterification processes. Bioresource Technology, v. 194, p. 179-186, out. 2015. 
CHEN, G.; ZHAO, L.; QI, Y. Enhancing the productivity of microalgae cultivated in wastewater toward biofuel production: A critical review. Applied Energy, v. 137, p. 282-291, 1 jan. 2015.

CHINNASAMY, S., BHATNAGAR, A., CLAXTON, R., DAS, K. C. Biomass and bioenergy production potential of microalgae consortium in open and closed bioreactors using untreated carpet industry effluent as growth medium. Bioresource Technology, v. 101, n. 17, p. 6751-6760, set. 2010.

CHISTI, Y. Biodiesel from microalgae. Biotechnology Advances, v. 25, n. 3, p. 294-306, maio 2007.

CHRISTENSON, L.; SIMS, R. Production and harvesting of microalgae for wastewater treatment, biofuels, and bioproducts. Biotechnology Advances, v. 29, n. 6, p. 686-702, nov. 2011.

DASGUPTA, C. N., JOSE GILBERT, J., LINDBLAD, P., HEIDORN, T., BORGVANG, S. A., SKJANES, K., \& DAS, D. Recent trends on the development of photobiological processes and photobioreactors for the improvement of hydrogen production. International Journal of Hydrogen Energy, v. 35, n. 19, p. 1021810238 , out. 2010.

DE JESUS RAPOSO, M. F.; DE MORAIS, R. M. S. C.; DE MORAIS, A. M. M. B. Health applications of bioactive compounds from marine microalgae. Life Sciences, v. 93, n. 15 , p. 479-486, out. 2013.

DISMUKES, G. C., CARRIERI, D., BENNETTE, N., ANANYEV, G. M., POSEWITZ, M. C.. Aquatic phototrophs: efficient alternatives to land-based crops for biofuels. Current Opinion in Biotechnology, v. 19, n. 3, p. 235-240, jun. 2008.

DOSHI, A., PASCOE, S., COGLAN, L., \& RAINEY, T. J. Economic and policy issues in the production of algaebased biofuels: A review. Renewable and Sustainable Energy Reviews, v. 64, p. 329-337, out. 2016.

DU, Z., MA, X., LI, Y., CHEN, P., LIU, Y., LIN, X., RUAN, R. Production of aromatic hydrocarbons by catalytic pyrolysis of microalgae with zeolites: Catalyst screening in a pyroprobe. Bioresource Technology, v. 139, p. 397401, jul. 2013.

ESCORSIM, A. M.; DA ROCHA, G.; VARGAS, J. V. C.; MARIANO, A. B.; RAMOS, L. P.; CORAZZA, M. L.; CORDEIRO, C. S. Extraction of Acutodesmus obliquus lipids using a mixture of ethanol and hexane as solvent. Biomass and Bioenergy, v. 108, p. 470-478, out. 2018.

FANG, Y. R.; YEH, Y.; LIU, H. S. A novel strategy of biodiesel production from wet microalgae by direct saponification-esterification conversion (DSEC). Journal of the Taiwan Institute of Chemical Engineers, v. 83, p. 23-31, 2018.

FENTON, O.; Ó HUALLACHÁIN, D. Agricultural nutrient surpluses as potential input sources to grow third generation biomass (microalgae): A review. Algal Research, v. 1, n. 1, p. 49-56, maio 2012.

FRANCHINO, M., COMINO, E., BONA, F., RIGGIO, V. A. Growth of three microalgae strains and nutrient removal from an agro-zootechnical digestate. Chemosphere, v. 92, n. 6, p. 738-744, jul. 2013.

FRANCO, A. L. C., LÔBO, I. P., CRUZ, R. S. DA, TEIXEIRA, C. M. L. L., ALMEIDA NETO, J. A. DE, \& MENEZES, R. S. Biodiesel de microalgas: avanços e desafios. Química Nova, v. 36, n. 3, p. 437-448, 2013.
GERARDO, M. L., VAN DEN HENDE, S., VERVAEREN, H., COWARD, T., \& SKILL, S. C. Harvesting of microalgae within a biorefinery approach: A review of the developments and case studies from pilot-plants. Algal Research, v. 11, p. 248-262, set. 2015.

GOUVEIA, L., GRAÇA, S., SOUSA, C., AMBROSANO, L., RIBEIRO, B., BOTREL, E. P., SILVA, C. M. Microalgae biomass production using wastewater: Treatment and costs: Scale-up considerations. Algal Research, v. 16, p. 167-176, jun. 2016.

GREENWELL, H. C., LAURENS, L. M. L., SHIELDS, R. J., LOVITT, R. W., \& FLYNN, K. J. Placing microalgae on the biofuels priority list: a review of the technological challenges. Journal of The Royal Society Interface, v. 7, n. 46, p. 703-726, maio 2010.

HALIM, R.; DANQUAH, M. K.; WEBLEY, P. A. Extraction of oil from microalgae for biodiesel production: A review. Biotechnology Advances, v. 30, n. 3, p. 709-732, maio 2012.

HUANG, J., QU, X., WAN, M., YING, J., LI, Y., ZHU, F., LI, $\mathrm{W}$.. Investigation on the performance of raceway ponds with internal structures by the means of CFD simulations and experiments. Algal Research, v. 10, p. 64-71, jul. 2015.

JIANG, L., LUO, S., FAN, X., YANG, Z., GUO, R.. Biomass and lipid production of marine microalgae using municipal wastewater and high concentration of $\mathrm{CO} 2$. Applied Energy, v. 88, n. 10, p. 3336-3341, out. 2011.

KONG, Q., LI, L., MARTINEZ, B., CHEN, P., \& RUAN, R. Culture of Microalgae Chlamydomonas reinhardtii in Wastewater for Biomass Feedstock Production. Applied Biochemistry and Biotechnology, v. 160, n. 1, p. 9-18, 9 jan. 2010.

KUMAR, K., MISHRA, S. K., SHRIVASTAV, A., PARK, M. S., \& YANG, J.-W. Recent trends in the mass cultivation of algae in raceway ponds. Renewable and Sustainable Energy Reviews, v. 51, p. 875-885, nov. 2015.

LAAMANEN, C. A.; ROSS, G. M.; SCOTT, J. A. Flotation harvesting of microalgae. Renewable and Sustainable Energy Reviews, v. 58, p. 75-86, maio 2016.

LAM, M. K.; LEE, K. T. Microalgae biofuels: A critical review of issues, problems and the way forward. Biotechnology Advances, v. 30, n. 3, p. 673-690, maio 2012.

LI, Y., CHEN, Y.-F., CHEN, P., MIN, M., ZHOU, W., MARTINEZ, B., RUAN, R. Characterization of a microalga Chlorella sp. well adapted to highly concentrated municipal wastewater for nutrient removal and biodiesel production. Bioresource Technology, v. 102, n. 8, p. 5138-5144, 1 abr. 2011.

LI, Y., HORSMAN, M., WU, N., LAN, C. Q., \& DUBOISCALERO, N. Biofuels from Microalgae. Biotechnology Progress, v.24, p.815-820, 2008.

LORENZEN, J.; IGL, N.; TIPPELT, M.; STEGE, A.; QOURA, F.; SOHLING, U.; BRÜCK, T. Extraction of microalgae derived lipids with supercritical carbon dioxide in an industrial relevant pilot plant. Bioprocess and Biosystems Engineering, v. 40, n. 6, p. 911-918, 2017.

LYND, L. R., LASER, M. S., BRANSBY, D., DALE, B. E., DAVISON, B., HAMILTON, R., WYMAN, C. E.. How biotech can transform biofuels. Nature Biotechnology, v. 26, n. 2, p. 169-172, 1 fev. 2008.

MATA, T. M.; MARTINS, A. A.; CAETANO, N. S. Microalgae for biodiesel production and other 
applications: A review. Renewable and Sustainable Energy Reviews, v. 14, n. 1, p. 217-232, jan. 2010.

MOHN, F. H. Experiences and strategies in the recovery of biomass from mass cultures of microalgae. Algae biomass : production and use, Munich, Germany, 1980.

MOLINA GRIMA, E., BELARBI, E.-H., ACIÉN FERNÁNDEZ, F., ROBLES MEDINA, A., \& CHISTI, Y. Recovery of microalgal biomass and metabolites: process options and economics. Biotechnology Advances, v. 20, n. 7-8, p. 491-515, jan. 2003.

MOREIRA, M. Novas tecnologias de colheita de microalgas para a produção de biodiesel Novas tecnologias de colheita de microalgas para a produção de biodiesel Dissertação (Mestrado Integrado em Engenharia da Energia e do Ambiente) Universidade de Lisboa, Lisboa, Portugal. 2013.

NAIK, S. N., GOUD, V. V., ROUT, P. K., \& DALAI, A. K. Production of first and second generation biofuels: A comprehensive review. Renewable and Sustainable Energy Reviews, v. 14, n. 2, p. 578-597, fev. 2010.

PATIL, P. D.; DANDAMUDI, K. P. R.; WANG, J.; DENG, Q.; DENG, S. Extraction of bio-oils from algae with supercritical carbon dioxide and co-solvents. Journal of Supercritical Fluids, v. 135, p. 60-68, dez. 2018.

PATIL, V.; TRAN, K.-Q.; GISELRØD, H. R. Towards sustainable production of biofuels from microalgae. International journal of molecular sciences, v. 9, n. 7, p. 1188-95, jun. 2008.

PEQUENO, M. A. G. Avaliação do potencial do óleo da microalga cultivada chlorella sp. por cromatografia gasosa. Revista Analytica, v.57, p. 54, Fev. 2012.

PEREIRA, C. M. P., HOBUSS, C. B., MACIEL, J. V., FERREIRA, L. R., DEL PINO, F. B., MESKO, M. F., COLEPICOLO NETO, P.. Biodiesel renovável derivado de microalgas: avanços e perspectivas tecnológicas. Química Nova, v. 35, n. 10, p. 2013-2018, 2012.

POHNDORF, R. S., CAMARA, Á. S., LARROSA, A. P. Q., PINHEIRO, C. P., STRIEDER, M. M., \& PINTO, L. A. A. Production of lipids from microalgae Spirulina sp.: Influence of drying, cell disruption and extraction methods. Biomass and Bioenergy, v. 93, p. 25-32, out. 2016.

PRAGYA, N.; PANDEY, K. K.; SAHOO, P. K. A review on harvesting, oil extraction and biofuels production technologies from microalgae. Renewable and Sustainable Energy Reviews, v. 24, p. 159-171, ago. 2013.

PRAVEENKUMAR, R., KIM, B., CHOI, E., LEE, K., PARK, J.-Y., LEE, J.-S., OH, Y.-K.. Improved biomass and lipid production in a mixotrophic culture of Chlorella sp. KR1 with addition of coal-fired flue-gas. Bioresource Technology, v. 171, p. 500-505, nov. 2014.

RANGEL-YAGUI CDE O1, DANESI ED, DE CARVALHO JC, SATO S.. Chlorophyll production from Spirulina platensis: cultivation with urea addition by fed-batch process. Bioresource Technology, v. 92, p. 133-141, 2004.

RASHID, N., UR REHMAN, M. S., SADIQ, M., MAHMOOD, T., \& HAN, J.-I. Current status, issues and developments in microalgae derived biodiesel production. Renewable and Sustainable Energy Reviews, v. 40, p. 760-778, dez. 2014.

RAWAT, I., RANJITH KUMAR, R., MUTANDA, T., \& BUX,
F. Biodiesel from microalgae: A critical evaluation from laboratory to large scale production. Applied Energy, v. 103, p. 444-467, mar. 2013.

RAWAT, I., RANJITH KUMAR, R., MUTANDA, T., \& BUX, F. Dual role of microalgae: Phycoremediation of domestic wastewater and biomass production for sustainable biofuels production. Applied Energy, v. 88, n. 10, p. 3411-3424, out. 2011.

RIZWAN, M.; LEE, J. H.; GANI, R. Optimal design of microalgae-based biorefinery: Economics, opportunities and challenges. Applied Energy, v. 150, p. 69-79, jul. 2015.

RODRIGUES, D. B., FLORES, É. M. M., BARIN, J. S., MERCADANTE, A. Z., JACOB-LOPES, E., \& ZEPKA, L. Q. Production of carotenoids from microalgae cultivated using agroindustrial wastes. Food Research International, v. 65, p. 144-148, nov. 2014.

RODRIGUES, R. T. J. RUBIO (2007). DAF-dissolved air flotation: Potential applications in the mining and mineral processing industry. International Journal of Mineral Processing v. 82, n. 1, p. 1-13, 2007.

SANDER, K.; MURTHY, G. S. Life cycle analysis of algae biodiesel. The International Journal of Life Cycle Assessment, v. 15, n. 7, p. 704-714, ago. 2010.

SANTOS,M.M. Novas tecnologias de colheita de microalgas para a produção de biodiesel. 2013.60f. Dissertação (Mestrado Integrado em Engenharia da Energia e do Ambiente) - Departamento de Engenharia Geográfica, Geofísica e Energia, Universidade de Lisboa, Lisboa. 2013.

SEN GUPTA, S.; SHASTRI, Y.; BHARTIYA, S. Model-based optimisation of biodiesel production from microalgae. Computers \& Chemical Engineering, v. 89, p. 222-249, jun. 2016.

SEYED HOSSEINI, N.; SHANG, H.; SCOTT, J. A. Optimization of microalgae-sourced lipids production for biodiesel in a top-lit gas-lift bioreactor using response surface methodology. Energy, v. 146, p. 47-56, 2018.

SHOMAL, R.; HISHAM, H.; MLHEM, A.; HASSAN, R.; ALZUHAIR,. Simultaneous extraction-reaction process for biodiesel production from microalgae. Energy Reports, v. 5, p. 37-40, nov. 2019.

SHOW, K.-Y. Algal Biomass Harvesting. Biofuels from Algae, p. 85-110, jan. 2014.

SIALVE, B.; BERNET, N.; BERNARD, O. Anaerobic digestion of microalgae as a necessary step to make microalgal biodiesel sustainable. Biotechnology Advances, v. 27, n. 4, p. 409-416, jul. 2009.

SILVE, A.; PAPACHRISTOU, I.; WÜSTNER, R.; STRÄSSNER, R.; SCHIRMER, M.; LEBER, K.; GUO, B.; INTERRANTE, L.; POSTEN, C.; FREY, W. Extraction of lipids from wet microalga Auxenochlorella protothecoides using pulsed electric field treatment and ethanol-hexane blends. Algal Research, v. 29, p. 212222, nov. 2018.

SINGH, V.; TIWARI, A.; DAS, M. Phyco-remediation of industrial waste-water and flue gases with algal-diesel engenderment from micro-algae: A review. Fuel, v. 173, p. 90-97, jun. 2016.

SUGANYA, T., VARMAN, M., MASJUKI, H. H., \& RENGANATHAN, S.. Macroalgae and microalgae as a potential source for commercial applications along with biofuels production: A biorefinery approach. Renewable 
and Sustainable Energy Reviews, v. 55, p. 909-941, mar. 2016

TOMMASI, E.; CRAVOTTO, G.; GALLETTI, P.; GRILLO, G.; MAZZOTTI, M.; SACCHETTI, G.; SAMORÌ, C.; TABASSO, S.; TACCHINI, M.; TAGLIAVINI, E. Enhanced and Selective Lipid Extraction from the Microalga P. tricornutum by Dimethyl Carbonate and Supercritical CO2 Using Deep Eutectic Solvents and Microwaves as Pretreatment. ACS Sustainable Chemistry and Engineering, v. 5, n. 9, p. 8316-8322, 2017.

UDUMAN, N., QI, Y., DANQUAH, M. K., \& HOADLEY, A. F. A. Marine microalgae flocculation and focused beam reflectance measurement. Chemical Engineering Journal, v. 162, n. 3, p. 935-940, set. 2010.

VANDAMME, D.; FOUBERT, I.; MUYLAERT, K. Flocculation as a low-cost method for harvesting microalgae for bulk biomass production. Trends in Biotechnology, v. 31, n. 4, p. 233-239, abr. 2013.

VERMA, P.; SHARMA, M. P. Review of process parameters for biodiesel production from different feedstocks. Renewable and Sustainable Energy Reviews, v. 62, p. 1063-1071, set. 2016.

VIGUERA, M., MARTI, A., MASCA, F., PRIETO, C., \& CALVO, L.. The process parameters and solid conditions that affect the supercritical $\mathrm{CO} 2$ extraction of the lipids produced by microalgae. The Journal of Supercritical Fluids, v. 113, p. 16-22, 1 jul. 2016.

VINOTH ARUL RAJ, J.; BHARATHIRAJA, B.; VIJAYAKUMAR, B.; AROKIYARAJ, S.; IYYAPPAN, J.; PRAVEEN KUMAR, R. Biodiesel production from microalgae Nannochloropsis oculata using heterogeneous Poly Ethylene Glycol (PEG) encapsulated ZnOMn2+ nanocatalyst. Bioresource Technology, v. 282, n. March, p. 348-352, jun. 2019.

WAN MAHMOOD, W. M. A.; THEODOROPOULOS, C.; GONZALEZ-MIQUEL, M. Enhanced microalgal lipid extraction using bio-based solvents for sustainable biofuel production. Green Chemistry, v. 19, n. 23, p. 5723-5733, 2017.

YEN, H.-W., HU, I.-C., CHEN, C.-Y., HO, S.-H., \& LEE, D.J.. Microalgae-based biorefinery - From biofuels to natural products. Bioresource Technology, v. 135, p. 166-174, 1 maio 2013.

ZHANG, Y.; KONG, X.; WANG, Z.; SUN, Y.; ZHU, S.; LI, L.; LV, P. Optimization of enzymatic hydrolysis for effective lipid extraction from microalgae Scenedesmus sp. Renewable Energy, v. 125, p. 1049-1057, 2018. 